\title{
Drug use problems with self-injected low-molecular-weight heparins in primary care
}

\author{
Seraina Mengiardi • Dimitrios A. Tsakiris • \\ Markus L. Lampert • Kurt E. Hersberger
}

Received: 12 August 2010 /Accepted: 10 November 2010 /Published online: 24 November 2010

(C) Springer-Verlag 2010

\begin{abstract}
Purpose Outpatient subcutaneous therapies are becoming increasingly common. A literature search failed to find produced any studies on application problems pertaining to the self-injection of low-molecular-weight heparins (LMWH) in a heterogeneous outpatient population under daily-life conditions. We therefore designed a study with the aim of recording drug use problems, patient satisfaction, compliance, problems arising from the injection site (abdomen vs. thigh), and residual drug volumes in prefilled syringes used in self-injection therapy.

Methods Patients were recruited in community pharmacies by 95 trained Master's students in pharmacy. Data were collected during recruitment and by means of structured questionnaire-based telephone interviews that were carried out at the beginning and the end of the LMWH treatment. Results The median age of the 213 patients enrolled in the study was 54 years [interquartile range (IQR) 39-70 years]; of these, $15.5 \%$ had their injections administered by a third person. The rate of self-reported non-compliance was $17.1 \%$. At least one relevant problem was recorded in
\end{abstract}

S. Mengiardi $(\bowtie) \cdot$ K. E. Hersberger

Pharmaceutical Care Research Group, Pharmacenter,

University of Basel,

Klingelbergstrasse 50,

4056, Basel, Switzerland

e-mail: seraina.mengiardi@unibas.ch

D. A. Tsakiris

Division of Hematology, University Hospital Basel,

Spitalstrasse 21/Petersgraben 4 ,

4031, Basel, Switzerland

M. L. Lampert

Clinical Pharmacy, Kantonsspital Bruderholz,

4101, Bruderholz, Switzerland
$85.0 \%$ of the cases. At the end of the treatment, $38.9 \%$ of the patients stated self-administration of the injections required some effort. The preferred injection site was the thigh $(68.5 \%)$. An overall mean residual drug volume $\geq 10.0 \%$ was detected for $3.9 \%$ of the patients. If residual drug was present, a median of $11.2 \%$ (IQR $8.6-$ $17.6 \%)$ of the total drug volume had not been injected. Patients injecting into the thigh showed a higher risk of leaving residual medication (odds ratio $2.16,95 \%$ confidence interval 1.04-4.51).

Conclusions Most patients had drug use problems, whereas no clear factors were associated with non-compliance, the injection site (apart from residual drug), and discomfort or effort required (apart from prior injection use).

Keywords Low-molecular-weight heparin · Outpatients · Drug use problems · Subcutaneous injections .

Injection site - Community pharmacy

\section{Introduction}

Low-molecular-weight heparins (LMWH) are frequently used for the prevention and treatment of venous thromboembolism [1-3]. There is strong evidence demonstrating the good benefit-to-risk ratio and cost-effectiveness of venous thromboembolism prophylaxis [1]. Treatments with LMWH are often started during a hospital stay or at hospital discharge and followed up by daily subcutaneous (s.c.) self-injections in an ambulatory setting for a period of time varying from days to weeks. Results from published studies demonstrate that home treatment of deep vein thrombosis with LMWH is at least as safe and effective as inpatient treatment-and may save costs and increase patient satisfaction $[4,5]$. 
Approaches involving outpatient s.c. therapies for the treatment of different diseases are becoming increasingly common. In addition to being used for the injection of the LMWH, pre-filled ready-to-use syringes are readily available for the treatment of multiple sclerosis (e.g., interferons), arthritis (e.g., methotrexate, tumor necrosis factor alpha blocker), anemia (e.g., erythropoietin), cancer (e.g., interferons), female infertility (hormones), hepatitis B and C (e.g., interferons) as well as for contraception (medroxyprogesterone acetate). Additional devices are pens, which are used by diabetic patients (insulin, exenatide) or for migraine treatment (e.g., sumatriptan), injectors, which are often used in the treatment of osteoporosis (recombinant parathyroid hormone analogue), or vials/ampules, where preparation is needed before injection (e.g., female infertility, cancer, multiple sclerosis and enfuvirtide in human immunodeficiency virus treatment). A search of the literature failed to identify studies focusing on drug use problems and/or the practical aspects of s.c. self-administration beside the LMWH in an outpatient setting. Rather, most of the studies on the self-injection of other agents concentrated on other aspects of this therapeutic approach, such as pharmacokinetics, effectiveness, safety, and patient satisfaction.

Discussions on the preferred injection site are ongoing, especially with LMWH [6-9]. Case reports of hematomas in the abdominal wall and rectus sheath due to s.c. injections into the abdominal wall are rare, but appear regularly in the literature [10-18]. Risk factors seem to be advanced age, female gender, polymorbidity, renal impairment, cough, therapeutic LMWH dosages, and concomitant use of anticoagulants. There is no expert consensus on the preferable injection site, often not even within one hospital. Patients who have already received LMWH treatment in the past are especially irritated when they receive a complete new set of instructions. Even more confusing is the wording for the abdomen and thigh injection sites: for example, eight different terms pertaining to the abdomen and five descriptions of the injection in the thigh were found in Swiss package inserts and leaflets. Expressions such as "ventral, collateral region of the abdomen" or "outer upside of the thigh" are difficult to visualize, especially by the layperson. The injection sites "back of the upper arm" or the "gluteal area" are rarely used, as these sites are unsuitable for self-injections. In addition to the injection site, a proper injection technique contributes to a safe and positive outcome, i.e., injecting slowly into a skin fold to reduce site pain and bruising $[19,20]$ as well as to ensuring that the injection is subcutaneous and not intramuscular.

Little information on drug use problems and compliance with LMWH treatment in outpatients is available in the literature [21-24]. Previous studies only investigated orthopedic patients recruited from selected clinics or hospitals. All of these study participants received educa- tional programs that included instruction in the injection technique, performing their first self-injection in the presence of a medical professional (nurse or physician), and (occasionally) written information material or a video tape. Study sizes ranged from 40 to 214 patients. However, we were unable to find any study involving a heterogeneous patient population receiving standard care.

We therefore designed a prospective cross-sectional study using pharmacy customers treated with LMWH as a convenient representative population receiving s.c. therapies with pre-filled syringes under daily life conditions. Our aim was to record drug use problems, patient satisfaction, and patient compliance. The results should highlight potential areas for improvements in patient care through specific interventions. The secondary aims were to identify differences in problems arising due to the choice of injection site (abdomen vs. thigh) and to determine residual drug volumes in the used syringes.

\section{Methods}

Setting and study population

Patients were recruited sequentially in community pharmacies by pharmacy students during their internship. Between January and May 2008, 95 Master's students of the two German-speaking universities of Basel and Zurich were instructed to recruit and interview ambulatory LMWH patients. In advance, the students received: (1) a detailed oral study briefing and written information; (2) documents for data collection; (3) instructions in the s.c. injection technique, including clinical training by nursing staff.

A broad range of inclusion criteria was deliberately chosen with the intention of reaching a varied sample of LMWH patients reflecting all aspects of daily life: outpatients aged $\geq 18$ years, all brands of LMWH (pre-filled syringes), prophylactic or therapeutic use, new or long-term prescription, first or previous outpatient s.c. treatment, all therapy durations, self-injection or application by another person (e.g. family member, nursing service), and no comprehension difficulties due to language.

\section{Data collection}

Routine prescription validation by each community pharmacy (standard care) was performed when a LMWH was requested. The study was explained to the person bringing the prescription (the patients themselves or another person) and instructions were given on the s.c. injection technique if required. If the patient met the inclusion criteria and oral consent was obtained, written patient information and a sharps collector (E-safe) for the used syringes were delivered. 
Telephone interview

At a pre-arranged date-either 1-3 days after the prescription was filled or at start of the LMWH treatment - an extensive structured questionnaire-based telephone interview was carried out. The trained students filled in the questionnaire by interviewing only the patient, even if the injections were carried out by another person. The survey consisted of open questions wherever possible, and patients' spontaneous answers were recorded. Multiple answers were accepted, but no answer suggestions were allowed. The reason for carrying out this telephone interview at an early point in the LMWH treatment was to evaluate drug use problems, the amount of effort required to self-inject, and discomfort at the beginning of the treatment. Self-estimations were assessed on two different scales: (1) an 11-point scale to rate discomfort $(0=$ very uncomfortable; $10=$ very comfortable) and (2) a 4-point scale to assess the degree of effort required $(1=$ no effort required at all; $2=$ nearly no effort required; $3=$ sometimes effort required; $4=$ considerable effort required) and drug use problems in general. In addition, the interview gathered information on patient and medication characteristics, selfmanagement, knowledge, quality of care, and patient satisfaction. If patients confirmed being impaired in their daily activities due to any kind of problem, pain, injury, or illness associated with the arm, shoulder, or hand, we rated the patient as being impaired in fine motor skills.

\section{Final interview}

After completion of the s.c. therapy, a short, structured questionnaire-based interview was carried out with each patient when he/she returned the sharps collector to their community pharmacy for professional disposal. The questions focused on the amount of effort required to maintain the treatment (none at any time; only in the beginning of treatment; occasionally; during the whole treatment period), on discomfort at the end of treatment, and on self-reported non-compliance. Exactly when this short interview took place depended on each individual's treatment duration. Patients were instructed to return their sharps collector after 6 weeks if the treatment period was longer.

The data collection was anonymized by assigning a code to each patient. Participants were asked to give oral consent each time they were contacted. The study protocol was approved by the local Ethics Committee of Basel (EKBB 95/07).

\section{Analysis of used syringes}

The returned sharps collectors were examined for the following: identification of patient code and syringe type; number of used syringes; number of syringes with recapping (illegitimate replacement of the needle shieldsometimes called needle cap-after injection); number of syringes with a visible residual drug volume; amount of residual drug volume; number of syringes with a correctly installed safety device for the prevention of needle stick injuries after injection.

Because the residual drug had often evaporated (especially in syringes without recapping), its volume could only be reliably determined by measuring the distance between the plunger and the end of the syringe barrel. Accordingly, the residual volume could be estimated by comparison with an unused syringe of the same type (taking the air bubble into consideration). To obtain this reference distance, we calculated the mean values of at least three syringes of each type. The mean residual drug volume (as a percentage) was defined as being equal to the calculated mean residual distance (percentage). Unused syringes were not included in this analysis. We considered a residual drug volume to be relevant if $\geq 10.0 \%$ of the total volume remained in the syringe.

\section{Statistical analysis}

The interview data sheets were processed with the automated form-processing software TELEform ver. 10.2 (Cardiff Software, Vista CA). To avoid potential errors, we verified the data transfer by visually comparing the written sheet and on-screen data. All data were then checked for plausibility by the first author. Free-text answers and comments were recorded separately and grouped during the plausibility-process by the first author. Missing data were complemented in the database according to the annotations if possible. Statistical analysis was performed using SPSS for Windows ver. 17.0 (SPSS, Chicago, IL).

In the descriptive analysis, medians and interquartile ranges (IQR; 25th to 75th percentile) were calculated. Pearson's chi-square test was used to investigate possible associations between two variables in a four-fold table. For unrelated group analyses, the non-parametric tests MannWhitney and Kruskall-Wallis were chosen. Analog tests for normal distribution [Student's $t$ test, analysis of variance (ANOVA)] were employed if the results differed. Statistical significance was set at $p \leq 0.05$.

\section{Results}

Of the 402 people approached by the pharmacy students when they went to a community pharmacy with a prescription for LMWH, 223 agreed to participate in the study and 144 completed the study (Fig. 1). Drop-outs did not differ from study completers in terms of age $(p=0.37$, 
Fig. 1 Study flowchart with numbers of patients and reasons for dropout. $L M W H$ Low-molecular-weight heparins

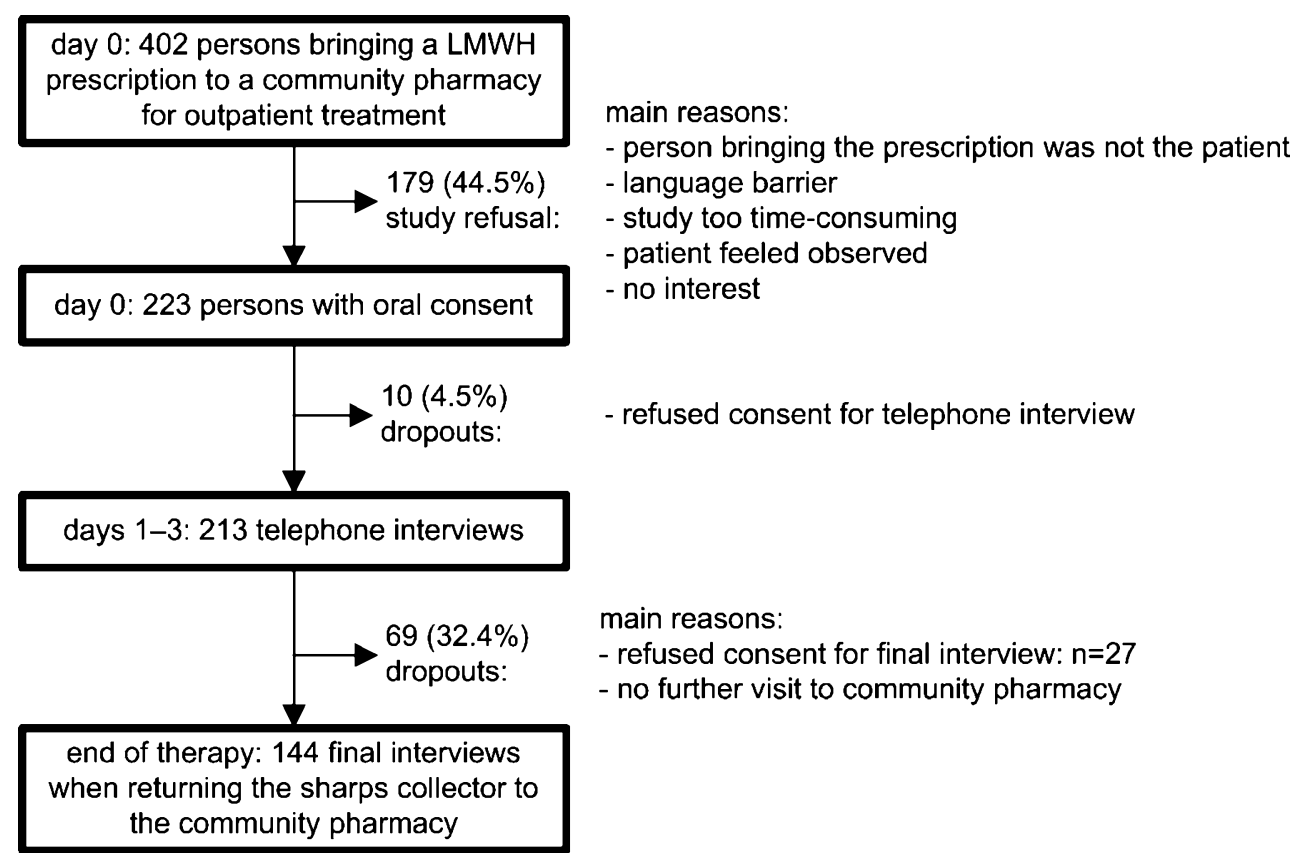

Mann-Whitney test), sex ( $p=0.93$, chi-square test), previous outpatient s.c. injection therapies ( $p=0.76$, chi-square test), injections administered by another person $(p=0.06$, chi-square test), little instruction (no oral instruction in the injection technique or only by the pharmacy; $p=0.66$, chi-square test), the degree of effort required ( $p=0.56$, Mann-Whitney test), discomfort ( $p=0.91$, Mann-Whitney test), or fine motor skills $(p=0.40$, chi-square test). Patient and medication characteristics are listed in Table 1.

Table 2 summarizes patients' self-reports on application problems, self-management, knowledge, non-compliance, and quality of care experienced (including patient satisfaction). We defined drug use problems to be relevant when: (1) patients were insufficiently informed about the injection site or technique; (2) injections were administered by another person; (3) recapping was carried out; (4) difficulties with removal of the needle shield existed; (5) there were discrepancies with prescribed therapy duration, daily injections, and injection time. At least one of these problems was reported in $181(85.0 \%)$ patients. The community pharmacy instructed $10.8 \%$ of the patients in the injection technique.

Self-reported non-compliance showed no association with age ( $p=0.85$, Mann-Whitney test), previous outpatient s.c. injection therapies ( $p=0.94$, chi-square test), injections administered by another person ( $p=0.18$, chi-square test), the degree of effort required ( $p=0.53$, Mann-Whitney test), little instruction ( $p=0.23$, chi-square test), discomfort ( $p=$ 0.15 , Mann-Whitney test), or fine motor skills $(p=0.24$, chi-square test). No significant associations were seen between the estimations of effort required and discomfort experienced with the variables first self-injection under the supervision of a medical professional $(p=0.62$ and 0.56 , respectively; Mann-Whitney test), little instruction ( $p=0.66$ and 0.22 , respectively; Mann-Whitney test), injections administered by another person ( $p=0.32$ and 0.83 , respectively; Mann-Whitney test), or the injection sites abdomen versus thigh ( $p=0.60$ and 0.91 , respectively; Mann-Whitney test). Patients with experience gained from previous outpatient s.c. injection therapies had less discomfort and the injections required less effort ( $p=0.011$ and 0.022 , respectively; MannWhitney test). Comfort/confidence with the injections and the degree effort required showed a Spearmen's correlation coefficient of $r=-0.5(p<0.001)$.

Of the 144 patients completing in the study, 126 estimated their comfort and effort required at the beginning and at the end of treatment (18 patients did not assess these parameters at the final interview as the injections were administered by another person). At the beginning of the therapy, $75.4 \%$ estimated their confidence with selfinjecting as high (scale levels $8-10$ ), while $32.5 \%$ reported that the injection required some effort. At the end of treatment, the corresponding values were $81.7 \%$ and $38.9 \%$, respectively. Comfort and effort required did not change significantly over time $(p=0.08$ and 0.13 , respectively, McNemar test). Nine (7.1\%) persons stated that the injections required effort throughout treatment, resulting in complete non-compliance in one case. Ten of the 126 patients sometimes had their injections administered by another person.

A comparison of the abdomen and thigh injections sites revealed no significant associations between puncture ( $p=$ 0.14 , Mann-Whitney test) or injection ( $p=0.38$, MannWhitney test) being unpleasant or painful and the side 
Table 1 Characteristics of study sample $\left(n_{\text {total }}=213\right)$

\begin{tabular}{|c|c|c|}
\hline Patient and clinical characteristics & $n(\%)^{\mathrm{a}}$ & Missing data $n(\%)$ \\
\hline \multicolumn{3}{|l|}{ Patient characteristics } \\
\hline Age (years) & $54(39-70)$ & $2(0.9)$ \\
\hline Males & $108(50.7)$ & $2(0.9)$ \\
\hline Education: & & $14(6.6)$ \\
\hline mandatory school & $24(11.3)$ & \\
\hline skilled worker & $123(57.7)$ & \\
\hline technical college + university & $52(24.4)$ & \\
\hline Previous outpatient s.c. injection therapies & $89(41.8)$ & $1(0.5)$ \\
\hline Impairment in daily living due to arm, shoulder, or hand & $29(13.6)$ & $9(4.2)$ \\
\hline Arthritis in arm, shoulder, or hand & $32(15.0)$ & $20(9.4)$ \\
\hline Impaired vision (using glasses or contact lenses) & $27(12.7)$ & $26(12.2)$ \\
\hline \multicolumn{3}{|l|}{ Medication characteristics } \\
\hline Medication & & $0(0.0)$ \\
\hline Fragmin (dalteparin) & $99(46.5)$ & \\
\hline Fraxiparine (nadroparin) & $63(29.6)$ & \\
\hline Clexane (enoxaparin) & $33(15.5)$ & \\
\hline Fraxiforte (nadroparin) & $9(4.2)$ & \\
\hline Sandoparin (certoparin) & $9(4.2)$ & \\
\hline Application once daily & $171(80.3)$ & $1(0.5)$ \\
\hline Not specified on prescription & $27(12.7)$ & \\
\hline \multicolumn{3}{|c|}{ Concomitant medication with an increased bleeding risk (not necessarily on the same prescription) } \\
\hline Anticoagulant (acetylsalicylic acid, phenprocoumon, acenocoumarol, clopidogrel) & $68(31.9)$ & $4(1.9)$ \\
\hline anticoagulant stopped during LMWH treatment & $28 / 68(41.2)$ & $3 / 68(4.4)$ \\
\hline Prescribed analgesic & $146(68.5)$ & $7(3.3)$ \\
\hline only paracetamol & $37 / 146(25.3)$ & $0 / 146(0.0)$ \\
\hline Self-medication with analgesics: & $20(9.4)$ & $2(0.9)$ \\
\hline only paracetamol & $9 / 20(45.0)$ & $0 / 20(0.0)$ \\
\hline Reason for LMWH treatment (multiple answers possible) & & $0(0.0)$ \\
\hline \multicolumn{3}{|l|}{ Surgery/injury of } \\
\hline lower limb & $112(52.6)$ & \\
\hline hip & $11(5.2)$ & \\
\hline upper limb & $7(3.3)$ & \\
\hline Thrombosis, embolism & $35(16.4)$ & \\
\hline Perioperative management/bridging & $16(7.5)$ & \\
\hline Atrial fibrillation, myocardial infarction & $8(3.8)$ & \\
\hline Cancer & $7(3.3)$ & \\
\hline Pregnancy, hormone therapy & $6(2.8)$ & \\
\hline Abdominal surgery & $6(2.8)$ & \\
\hline Long-distance travel & $4(1.9)$ & \\
\hline Other & $12(5.6)$ & \\
\hline
\end{tabular}

s.c., Subcutaneous, LMWH, low-molecular-weight heparins

a All data is presented as the number $(n)$ with the percentage in parenthesis with the exception of 'Age', which is presented as the median with the interquartile range in parenthesis

effects hematoma ( $p=0.50$, chi-square test), mild injection site irritation ( $p=0.34$, chi-square test), and site pain ( $p=$ 0.24 , chi-square test).

When only patients who always or sometimes selfadministered the LMWH were considered $(n=187)$, signif- icant differences between the level of difficulty encountered in removing the needle shield were found between the different brands of syringes needle shield $(p=0.037$, Kruskal-Wallis test). Based on pairwise differences, the needle shield of Fragmin was rated as significantly easier to 
Table 2 Self-reported quality of care (including patient satisfaction), self-management, drug use problems, knowledge, and non-compliance $\left(n_{\text {total }}=213\right)$

\begin{tabular}{|c|c|c|}
\hline Parameters on patients' self-reports & $n(\%)^{\mathrm{a}}$ & Missing data $n$ \\
\hline \multicolumn{3}{|l|}{ Quality of care and patient satisfaction } \\
\hline \multicolumn{2}{|l|}{ Oral instruction in injection technique (previous and present treatment): } & \multirow[t]{3}{*}{$0(0.0)$} \\
\hline None & $10(4.7)$ & \\
\hline Only by the pharmacy & $8(3.8)$ & \\
\hline Insufficiently informed about injection site & $8(3.8)$ & $7(3.3)$ \\
\hline Insufficiently informed about injection technique & $14(6.6)$ & $9(4.2)$ \\
\hline Alcohol swab provided & $200(93.9)$ & $1(0.5)$ \\
\hline \multicolumn{3}{|l|}{ First self-injection in the presence of a medical professional: } \\
\hline Provided & $111(52.1)$ & $0(0.0)$ \\
\hline helpful & $97 / 111(87.4)$ & $12 / 111(10.8)$ \\
\hline Not provided, but desired & $15 / 102(14.7)$ & $17 / 102(16.7)$ \\
\hline \multicolumn{3}{|l|}{ Delivery of leaflet: } \\
\hline Provided & $41(19.2)$ & $8(3.8)$ \\
\hline helpful & $33 / 41(80.5)$ & $3 / 41(7.3)$ \\
\hline Not provided, but desired & 28/164 (17.1) & 22/164 (13.4) \\
\hline \multicolumn{3}{|l|}{ First injection administered by the pharmacist: } \\
\hline Provided & $0(0.0)$ & $13(6.1)$ \\
\hline Not provided, but desired & $9 / 200(4.5)$ & $42 / 200(21.0)$ \\
\hline \multicolumn{3}{|l|}{ All injections administered by the pharmacist: } \\
\hline Provided & $0(0.0)$ & $12(5.6)$ \\
\hline Not provided, but desired & $8 / 201(4.0)$ & $33 / 201(16.4)$ \\
\hline \multicolumn{3}{|l|}{ Delivery of sharps collector: } \\
\hline Provided & $203(95.3)$ & $0(0.0)$ \\
\hline helpful & $135 / 203(66.5)$ & $38 / 203(18.7)$ \\
\hline Not provided, but desired & $0 / 10(0.0)$ & $4 / 10(40.0)$ \\
\hline \multicolumn{3}{|l|}{ Injection training into a "phantom" (injection pillow): } \\
\hline Provided & $7(3.3)$ & $8(3.8)$ \\
\hline helpful & $6 / 7(85.7)$ & $1 / 7(14.3)$ \\
\hline Not provided, but desired & $10 / 198(5.1)$ & $33 / 198(16.7)$ \\
\hline \multicolumn{3}{|l|}{ Video tape: } \\
\hline Provided & $1(0.5)$ & $10(4.7)$ \\
\hline helpful & $0 / 1(0.0)$ & $0 / 1(0.0)$ \\
\hline Not provided, but desired & $13 / 202(6.4)$ & $33 / 202(16.3)$ \\
\hline \multicolumn{3}{|l|}{ Self-management (multiple answers possible) } \\
\hline Injection site & & $0(0.0)$ \\
\hline Thigh & $146(68.5)$ & \\
\hline Abdomen & $80(37.6)$ & \\
\hline Back of the upper arm & $2(0.9)$ & \\
\hline Other & $2(0.9)$ & \\
\hline Injections administered by another person (sometimes or always) & $33(15.5)$ & $0(0.0)$ \\
\hline by family member/friend & $25 / 33(75.8)$ & \\
\hline by medical professional & $9 / 33(27.3)$ & \\
\hline Reasons for not self-injecting: & & $5 / 33(15.2)$ \\
\hline needle phobia & $9 / 33(27.3)$ & \\
\hline fear of puncturing skin & $8 / 33(24.2)$ & \\
\hline severely disabled & $4 / 33(12.1)$ & \\
\hline family member is a medical professional & $3 / 33(9.1)$ & \\
\hline other & $8 / 33(24.2)$ & \\
\hline
\end{tabular}


Table 2 (continued)

\begin{tabular}{|c|c|c|}
\hline Parameters on patients' self-reports & $n(\%)^{\mathrm{a}}$ & Missing data $n(\%)$ \\
\hline Illegitimate recapping & $157(73.7)$ & $5(2.3)$ \\
\hline \multicolumn{3}{|l|}{ Application problems } \\
\hline Difficulties with removal of needle shield & $28(13.1)$ & $1(0.5)$ \\
\hline Puncture is unpleasant/painful & $105(49.3)$ & $3(1.4)$ \\
\hline Injection is unpleasant/painful & $113(53.1)$ & $6(2.8)$ \\
\hline Degree of effort required to inject (scale: $1-4$ ) & $2(1-3)$ & $5(2.3)$ \\
\hline Confidence/lack of discomfort (scale: $0-10$ ) & $9(7-10)$ & $26(12.2)$ \\
\hline Side effects (multiple answers possible): & $105(49.3)$ & $2(0.9)$ \\
\hline Hematoma at injection site & $79(37.1)$ & \\
\hline Mild injection site irritation/burning & $36(16.9)$ & \\
\hline Hematoma in general & $16(7.5)$ & \\
\hline Site pain & $15(7.0)$ & \\
\hline Exanthema & $4(1.9)$ & \\
\hline Bleeding tendency & $4(1.9)$ & \\
\hline Induration & $4(1.9)$ & \\
\hline Epistaxis & $2(0.9)$ & \\
\hline Other & $9(4.2)$ & \\
\hline no action taken by study participants & $77 / 105(73.3)$ & $13 / 105(12.4)$ \\
\hline met criteria for reporting an adverse event to regulatory authority & $1(0.5)$ (arm exanthema) & $0(0.0)$ \\
\hline \multicolumn{3}{|l|}{ Knowledge } \\
\hline Discrepancy with prescribed therapy duration & $9(4.2)$ & $4(1.9)$ \\
\hline Not specified on prescription & $59(27.7)$ & \\
\hline Discrepancy with prescribed daily injections & $3(1.4)$ & $3(1.4)$ \\
\hline Not specified on prescription & $27(12.7)$ & \\
\hline Discrepancy with prescribed injection time & $7(3.3)$ & $3(1.4)$ \\
\hline Not specified on prescription & $157(73.7)$ & \\
\hline Nescience of reason for LMWH treatment & $6(2.8)$ & $0(0.0)$ \\
\hline Nescience of potential interactions with NSAR & $158(74.2)$ & $2(0.9)$ \\
\hline Nescience of potential side effects & $116(54.5)$ & $2(0.9)$ \\
\hline \multicolumn{3}{|l|}{ Self-reported non-compliance (assessed at final interview with $n=144$ patients) } \\
\hline Difficulties with injecting the LMWH timely & $15(10.4)$ & $2(1.4)$ \\
\hline Applications exceeding $\pm 2 \mathrm{~h}$ of assigned injection time & $5(3.5)$ & $1(0.7)$ \\
\hline Skipping injections ( $n=146$; completion of database according to annotations): & $25(17.1)$ & $0(0.0)$ \\
\hline 1 time & $8 / 25(32.0)$ & $4 / 25(16.0)$ \\
\hline$>3$ times & $5 / 25(20.0)$ & \\
\hline Reason for skipping injections (multiple answers possible): & & $1 / 25(4.0)$ \\
\hline forgotten & $11 / 25(44.0)$ & \\
\hline early discontinuation & $6 / 25(24.0)$ & \\
\hline not being at home & $2 / 25(8.0)$ & \\
\hline other $^{\mathrm{b}}$ & $7 / 25(28.0)$ & \\
\hline
\end{tabular}

NSAR, Non-steroidal anti-rheumatics

${ }^{\text {a }}$ All data is presented as the number $(n)$ with the percentage in parenthesis with the exceptions of 'Degree of effort required to inject' and 'Confidence/lack of discomfort', which are presented as the median with the interquartile range in parenthesis

${ }^{\mathrm{b}}$ Injections every 2-3 days depending on appearance of leg pain; vomiting or abdominal pain; delayed filling of the prescription; skeptical towards LMWH; news coverage about contaminated heparins; injection required too much effort (complete non-compliance); dropping a syringe leading to an insufficient number of syringes 
remove than those of Clexane and Fraxiparine $(p=0.021$ and 0.003, respectively, Mann-Whitney test).

Post-injection needle guards were only found with Fraxiparine and Fraxiforte devices. The needle guards of all syringes in the sharps collectors were correctly positioned by $22(32.8 \%)$ of the 67 patients injecting Fraxiparine or Fraxiforte (missing data: $n=5) ; 24$ (35.8\%) patients activated the safety device only partly, and 21 $(31.3 \%)$ patients did not use the needle guards at all or not properly (the protective guard is only securely locked in place once a clicking sound is heard after sliding it over the needle).

The sharps collectors of 180 patients contained a total of 3,218 syringes (median 10.5, IQR 8-26; range 1-100) (Fig. 2). The pre-filled syringes had volumes of $0.2-1.0 \mathrm{ml}$ and distances between the plunger and the end of the syringe barrel of 17.0-38.4 mm (including the air bubble). An overall mean residual drug volume $\geq 10.0 \%$ was detected for seven $(3.9 \%)$ patients (median injection volume $0.6 \mathrm{ml})$. The highest overall mean residual drug volume was $17.9 \%$, and was recorded for a patient who had injected 13 syringes of $0.8 \mathrm{ml}$.

When only those syringes with residual amounts of LMWH were considered, a median of $11.2 \%$ (IQR $8.6-$ $17.6 \%$ ) of the liquid remained in the syringe. In other words, if their syringes were not empty, 58 (59.8\%) of the 97 persons affected had $\geq 10.0 \%$ of the total volume not injected (Fig. 3). Comparisons between these 58 patients and the remaining 122 participants showed no differences in age ( $p=0.61$, Mann-Whitney test), sex $(p=0.72$, chisquare test), fine motor skills $(p=0.53$, chi-square test), previous outpatient s.c. injection therapies $(p=0.74$, chisquare test), injections administered by another person ( $p=$ 0.48 ; chi-square test), injection volumes ( $p=0.53$, MannWhitney test), the different brands ( $p=0.09$, chi-square test),

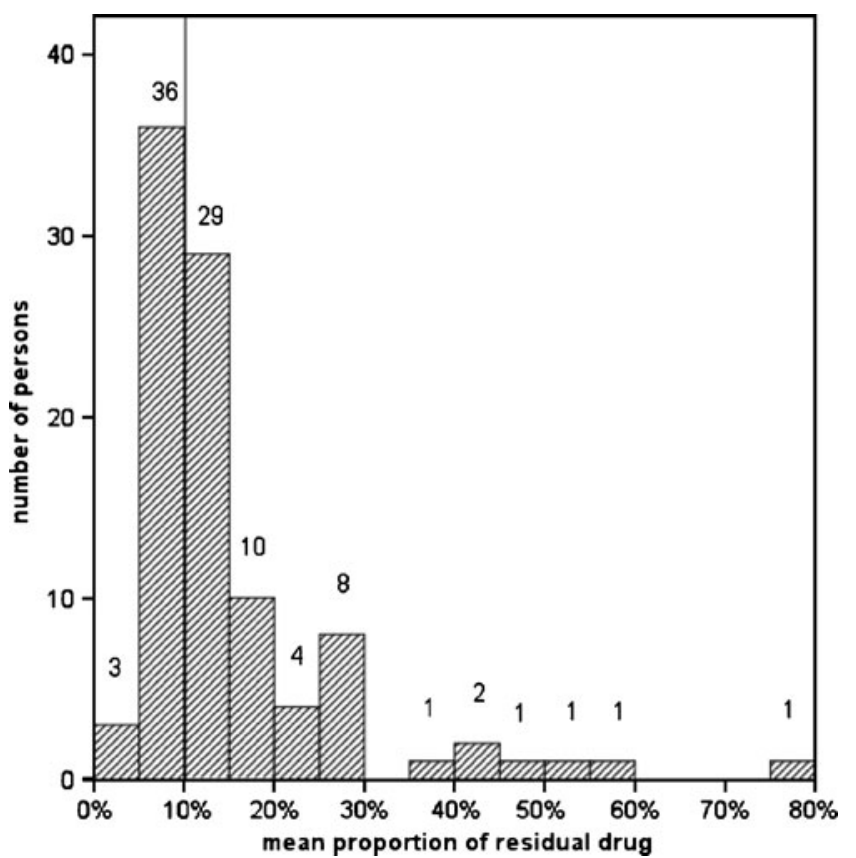

Fig. 3 Mean proportion of residual drug in used syringes still containing medication. Only those syringes with residual amounts of LMWH (97 patients, 304 syringes; range 1-16 syringes) were considered in the analysis

or number of used syringes as an indication of therapy duration ( $p=0.14$, Mann-Whitney test). However, these 58 patients injected significantly less into the abdomen $(p=$ 0.021 , chi-square test) and significantly more into the thigh $[p=0.019$, chi-square test; odds ratio 2.16 , $(95 \%$ confidence interval (CI) 1.04-4.51)].

Optional free-text comments provided deeper insights into the nature of the drug use problems. Handling difficulties were reported by 33 patients $(15.5 \%)$; the most important of these are listed in Table 3.

A student observed that the majority of his pharmacy customers' complaints were at the beginning of treatment.
Fig. 2 Prevalence of syringes with residual drug irrespective of the volume amount

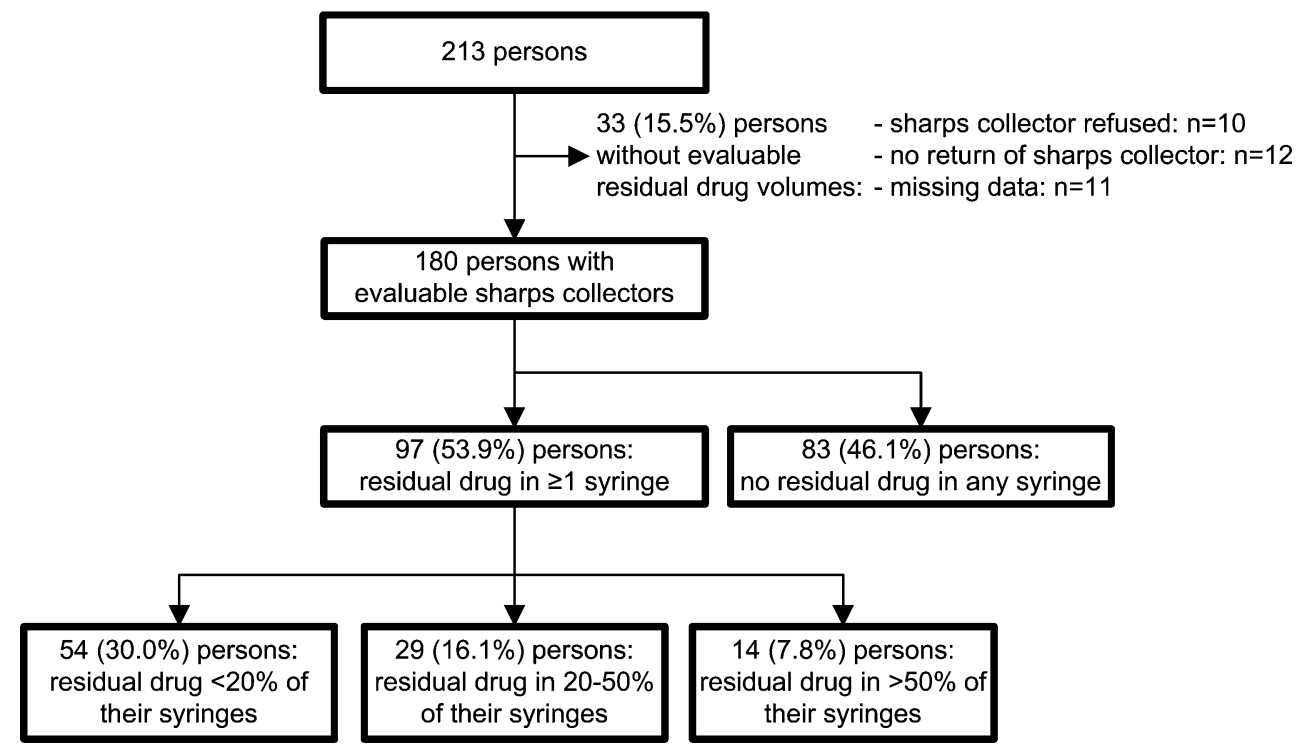


Table 3 Handling difficulties (multiple statements per person possible)

\begin{tabular}{ll}
\hline Component/action & Handling difficulty \\
\hline $\begin{array}{l}\text { Flap of paper backing on blister pack } \\
\text { Removal of needle shield }\end{array}$ & $\begin{array}{l}\text { Too small to remove the syringe from its packaging } \\
\text { Tricky; difficulties due to single-handed removal; bending the needle; total liquid loss due to pulling the } \\
\text { plunger rod } \\
\text { Too sharp; not sharp; twice blocked; bent }\end{array}$ \\
Needle & $\begin{array}{l}\text { Uncertainty whether air bubble needs to be removed; annoying; no air bubble } \\
\text { Air bubble }\end{array}$ \\
Injection & $\begin{array}{l}\text { concerning the insertion length of the needle into the skin; coordination difficult regarding quick insertion } \\
\text { of the needle vs. slow injection; high resistance when pushing the plunger rod in the beginning leading to } \\
\text { a sudden and quick injection; needle accidentally came out of the skin during injection; liquid loss during } \\
\text { first injection; early discontinuation due to lots of pain and problems during injection; injection by } \\
\text { another person, because of inability to self-inject into the back of the upper arm; setting back injection } \\
\text { time every day } 15 \text { min from } 7 \text { p.m. (injection time in hospital) to } 11 \text { p.m. (preferred injection time at home) } \\
\text { Needle stick injury; needle easily penetrates the soft needle shield } \\
\text { Syringe in general very small and hence difficult to handle ( } n=3) \text {; uncertainty whether total volume was } \\
\text { injected }(n=3) ; \text { dropping the syringe before injection }(n=2) \text {; finger flange too small }(n=2) ; \text { difficulties } \\
\text { with positioning the needle guard of Fraxiparine }\end{array}$ \\
$\begin{array}{l}\text { Recapping } \\
\text { Syringe }\end{array}$ &
\end{tabular}

In contrast, one patient's concerns increased towards the end of a 4-week treatment. Another person would have even changed from self-management to injections by another person if the therapy duration was longer than the actual 6 weeks. One patient injecting into the thigh had more side effects when injecting 0.6 than $0.4 \mathrm{ml}$.

Two patients showed restraint in injecting into the thigh; one chose the abdomen instead, and the second asked another person to administer the injection. One person noticed that hematoma generally developed more often when injecting into the abdomen.

The support offered was not always satisfying and highlights possible areas for improvement in patient care (Table 4).

\section{Discussion}

With respect to outpatient s.c. therapies, drug use problems appear to be very prevalent, diverse, and complex. They may be associated with the injection itself or with the handling of the injection-device. Notably, among the participants in our study, $85.0 \%$ experienced at least one relevant problem, with recapping being the most frequent difficulty encountered: $73.7 \%$ of the patients replaced the needle shields after injection, which is against recommended practice. At the end of the therapy, almost $40 \%$ reported that the injection required some effort, and $17.1 \%$ admitted non-compliance. Medical professionals are unable to ascertain potential patient problems in using medication at first glance. As a result, any outpatient s.c. therapy poses a challenge not only for the patients themselves and their family/friends, but also for health professionals. Therefore, adequate patient care and education are crucial and should be optimized.

In our study, $15.5 \%$ of patients had their injections performed by another person $(75.8 \%$ of these by family members or friends); of these, $51.5 \%$ of patients reported that this was due to needle phobia or a fear of puncturing the skin. A review of the literature shows a $13-37 \%$ nonself-injecting rate [21-24], and in 46.9-66.0\% of these cases family members administered the injections [21, 24]; $75.0 \%$ of those who refuse to self-inject report that it is due to fear [21]. This fact should be considered when designing patient education programs. If injections are given by a family member, this person should be properly instructed.

Table 4 Room for improvement in quality of care (multiple statements per person possible)

\begin{tabular}{ll}
\hline General improvements & Specific improvements \\
\hline $\begin{array}{l}\text { More information } \\
\text { Improved instruction in the }\end{array}$ & $\begin{array}{l}\text { On thromboembolism }(n=4) \text { and its prevention }(n=2) \text {; on LMWH and side effects }(n=2) \\
\text { injection technique }\end{array}$ \\
$\begin{array}{l}\text { Better instruction }(n=9) ; \text { increased patient involvement }(n=8) \text {; instructions not only orally but with } \\
\text { on the day before hospital discharge }(n=2) ; \text { repetition of the instructions when collecting their prescription }\end{array}$ \\
$\begin{array}{ll}\text { Consistent instructions } & \text { On injection angle }(n=3) \text {; injection site }(n=2) ; \text { skin fold; air bubble } \\
\text { Better leaflets } & \text { On terminology; font size; foreign languages }\end{array}$ \\
\hline
\end{tabular}


Consequently, physicians, hospitals, and community pharmacies should always be equipped with the latest leaflets.

There were no associations between patient characteristics and outcome measures with effort required or discomfort, with the exception of previous experience in self-injecting. A possible explanation for the absence of associations could be the heterogeneity of the study sample. Discomfort and effort required did not change notably over time. The level of comfort with the procedure was quite high in general, with one reason probably being the fact that $41.8 \%$ of the patients already had experience of outpatient s.c. injection therapies (this patient group was significantly more confident and felt less effort was needed).

Important differences concerning difficulties with the removal of the needle shield between different LMWH brands were observed, which confirms the results of a previous investigation [25]. Only one third of patients injecting Fraxiparine or Fraxiforte applied the needle guards of all their used syringes accurately, and one third did not use them at all or used them improperly. Ironically, the danger for needle stick injuries increases when the needle guard is positioned but not locked in position, as protection is assumed but not provided. There is certainly room for improvement in this area through better patient information and education, particularly as correctly fitting the guard requires considerable force and coordination and the mechanism is poorly marked. Clexane and Arixtra have pre-filled syringes equipped with new automatic safety devices; the protective shield is triggered when the syringe is empty. In addition to preventing needle stick injuries, the Clexane and Arixtra syringes ensure that the whole amount is injected. In our study, there were no patients prescribed with Arixtra, and the new Clexane device and the new Fragmin Needle-Trap were not yet on the market in Switzerland.

Whether additional drug use problems were also mentioned by the participant when he/she was answering the questions posed during the telephone interview depended on the participant's openness for further conversation. It can be assumed that these anecdotic application problems would have been noted more frequently if they were asked for systematically. An example is the single statement of one participant about having more side effects when injecting 0.6 than $0.4 \mathrm{ml}$ into the thigh, which confirms the results from another study [26]. Thus, it is likely that not all problems were revealed, and those that were may be more multifaceted than previously imagined.

Prescriptions were often incomplete with regards to therapy duration $(27.7 \%)$, number of daily injections $(12.7 \%)$, and injection time $(73.7 \%)$. Missing written information makes patient care demanding. The probably unintentional single under- or overdoses due to a shift in the time interval of $10-12 \mathrm{~h}$ in comparison to the prescribed injection time occurred at a sensitive and susceptible moment after hospital discharge or at the beginning of treatment in the ambulatory setting. It can be expected that this was a much more common occurrence than the five observed cases, as the injection time was not given by $73.7 \%$ of patients. As such time-shifts generally happen unknowingly, patients would not have mentioned it when assessing their own compliance.

Concomitant self-medication with non-steroidal analgesics did not seem to play an important role, although only $25.8 \%$ of the participants knew about potential drug interactions with LMWH. The participants also showed a lack of knowledge of potential side effects. Overall, the assistance provided was appreciated by patients, but the amount of help needs to be increased.

Two thirds of the patients injected into the thigh and one third into the abdomen. Associations with local side effects or the puncture hole and injection being painful were not significant. In additional free-text answers, however, a number of patients mentioned problems comparing the two injection sites. The literature also seems to be ambiguous on this point: a Brazilian study reported that hematomas were observed in $83.7 \%$ of patients and that the incidence of hematomas was higher if the LMWH injections were administered into the thigh [27]. In contrast, in a special series of patients following standard herniotomia, hematoma appeared in $25 \%$ of the cases when the patients injected into the abdomen and in $9 \%$ when they injected into the thigh [9]. Other investigations comparing local side effects of LMWH according to the two injection sites were not found. In a study with s.c. injections of enfuvirtide, injection site reactions were common but mild, and their incidence was higher with injections into the abdomen than into the thigh or arm [28]. Patients using a sumatriptan self-injector experienced more bleeding and local pain when injecting into the thigh compared to the gluteal area; only $15 \%$ preferred the thigh as injection site [29].

Every sixth person (17.1\%) admitted to having skipped injections. In similar studies, non-compliance rates of 4.5$28.3 \%$ with different definitions of non-compliance were found [21-24]. The main reason in the study of Spahn was forgetfulness $(94.1 \%)$, while $13.1 \%$ of the patients discontinued early; all patients younger than 20 years were classified as unreliable and compliance was dependent on whether injections were self-administered [21]. Our study showed a wider variety of reasons for non-compliance, with the most important being forgetfulness $(44.0 \%)$ and early discontinuation $(24.0 \%)$. We were unable to identify possible risk factors for self-reported non-compliance, possibly because only 25 patients actually admitted having skipped injections, or the diverse reasons for non-compliance. 
Our original objective of determining patients' taking compliance by comparing the number of used syringes with the prescribed therapy duration turned out to be impossible. In $36.2 \%$ of the final interviews, the date of the last injection was not provided, and in $27.7 \%$ and $12.7 \%$ of the prescriptions, respectively, therapy duration and application frequency were not specified. In addition, terms such as "treatment until complete mobilization/international normalized ratio twice in therapeutic range/next visit with physician" did not enable the date of the last injection to be estimated reliably. Furthermore, the prescription date did not necessarily correlate with the day of discharge or start of LMWH treatment. Similarly, a patient could be fully compliant despite a delay in filling the prescription asparticularly on weekends or public holidays - syringes are often dispensed by hospitals or physicians to ensure therapeutic coverage. For various reasons, all used syringes were not discarded into the sharps collector: delayed delivery of sharps collector, injection with physician during consultation, not being at home, flights, holidays, and delivery of syringes by the hospital or physician.

The determination of the residual drug volumes enabled us to partially objectify patients' compliance: residual drug volumes were found rather sporadically, and almost half of the patients had no residual drug in any syringe. The overall mean residual drug volume was low and negligible, but the total injection volume seemed to have an influence, possibly as a result of rising tissue resistance due to the injection of higher volumes. This has a particular impact when LMWH are used for the treatment of thromboembolisms as higher volumes are administered and patients are at greater risk. If residual drug was present, however, it tended to be of pharmacological relevance. It can be expected that some of these injections were stopped early on purpose.

Patients injecting into the thigh showed a higher risk of leaving residual volumes, which may be due to the smaller area of s.c. tissue in the thigh compared to the abdomen. We therefore recommend that patients injecting high volumes or injecting into the thigh be advised to monitor closely whether the syringe is empty and to be aware that they might need more force towards the end of the injection. Other risk factors for residual volumes could not be identified. Sufficient evidence was not collected on this aspect, probably because the therapy durations were mostly short, with a median of 10.5 syringes in the sharps collectors. It has also to be taken into account that almost half of the patients $(47.2 \%)$ had to inject only small volumes of $\leq 0.3 \mathrm{ml}$, and a minority of $9.4 \%$ injected volumes of $\geq 0.7 \mathrm{ml}$.

The strength of our study is the heterogeneous study population, covering a broad spectrum of drug use problems and reflecting daily life activities. Not only compar- atively healthy patients participated in our study (e.g., those with a foot injury, long-distance travelers), but also seriously ill persons, such as patients with pulmonary embolism, lung transplantations, or paraplegia. However, categorization of such a study population is difficult. The community pharmacies were distributed more or less throughout Switzerland, which ensured that possible regional differences in the quality of care on the part of the physicians, hospitals, and community pharmacies were taken into account.

The main limitations of our study are the data collection by 95 students, a consent rate of only $55.5 \%$, and a possible bias due to patient selection. Polymorbid or cumbersome pharmacy customers were less likely asked to take part in the study, whereas regular or pleasant customers were more often invited to participate. Furthermore, interested and motivated patients are more likely to participate in a study and to be more compliant, reflecting daily life in a much too positive way. Another weakness is the dropout rate of $32.4 \%$ at the final interview, setting constraints on the conclusions that could be drawn on self-reported noncompliance and the estimations of comfort or effort required in the course of the therapy duration. As no prescription duplicate was requested and data collection was anonymous, no retracing or access to medical history was possible. Therefore, the results are based on patients' self-reports only. Our determination of the residual drug volume by measuring the distance between plunger and the end of the cylindrical body was the most reliable measurement, but the approach has limitations: as the liquid had often evaporated, we were unable to recognize whether we were measuring only missing liquid or the missing liquid together with the air bubble. Thus, the results are only estimations, although they are helpful in providing an impression of the magnitude of the problem. Hence, the true mean residual drug volumes may even be smaller.

\section{Conclusion}

Low-molecular-weight heparins represent a good model for studying outpatient s.c. therapies in primary care. Among our patient cohort, $85.0 \%$ reported some relevant drug use problem, whereas no clear factors were associated with non-compliance, the injection site (beside residual drug), and discomfort or effort required (beside prior injection use). Around $4 \%$ of patients had a considerable mean residual drug volume $(\geq 10.0 \%)$ in their syringes, with a higher risk of leaving medication when injection was into the thigh. The challenge facing not only for pharmacists but all health professionals as well as the pharmaceutical industry (design of injection-device and instruction leaflets) 
is to successfully contribute to a successful therapy. From a patient's point of view, injections require some effort. Therefore, it can be imagined that injection-free therapies for patients on chronic antithrombotic therapy would be appreciated.

Acknowledgments We would like to thank the participating community pharmacies for their cooperation. We also thank the Master's students for data collection, the patients for participating in the study, Michael Mittag for his support in the statistical analysis, and Irene Vogel Kahmann, Raphaela von Grünigen, Joëlle Bader, and Paul Lavender for their valuable contributions.

Conflict of interest This study was supported by Pfizer AG through an unrestricted Investigator-Initiated Research Grant (IIRG).

\section{References}

1. Geerts WH, Bergqvist D, Pineo GF, Heit JA, Samama CM, Lassen MR, Colwell CW (2008) Prevention of venous thromboembolism: American College of Chest Physicians Evidence-Based Clinical Practice Guidelines (8th edition). Chest 133[Suppl]:381S$453 \mathrm{~S}$

2. Bates SM, Greer IA, Pabinger I, Sofaer S, Hirsh J (2008) Venous thromboembolism, thrombophilia, antithrombotic therapy, and pregnancy: American College of Chest Physicians EvidenceBased Clinical Practice Guidelines (8th edition). Chest 133 [Suppl]:844S-886S

3. Ansell J, Hirsh J, Hylek E, Jacobson A, Crowther M, Palareti G (2008) Pharmacology and management of the vitamin K antagonists: American College of Chest Physicians Evidence-Based Clinical Practice Guidelines (8th edition). Chest 133[Suppl 6]:160S-198S

4. Othieno R, Abu Affan M, Okpo E (2007) Home versus in-patient treatment for deep vein thrombosis. Cochrane Database Syst Rev (3): CD003076

5. Matsagas MI (2004) Outpatient treatment of venous thromboembolism using low molecular weight heparins. An overview. Int Angiol 23:305-316

6. Fahs PS, Kinney MR (1991) The abdomen, thigh, and arm as sites for subcutaneous sodium heparin injections. Nurs Res 40:204-207

7. Stäubli M, Suter J (2004) Die Komplikationenliste der Schweizerischen Gesellschaft für Innere Medizin. Schweiz Aerztezeitung 85:11091116

8. Huber AR (2005) Fragen aus der Praxis zum Thema Antikoagulation. Prim Care 5:773

9. Lemke H, Imhoff M, Lohlein D (1994) Increased wound healing disorders in patients with inguinal hernia caused by administration of antithrombotic agents in the abdominal wall. Chirurg 65:714716

10. Donaldson J, Knowles CH, Clark SK, Renfrew I, Lobo MD (2007) Rectus sheath haematoma associated with low molecular weight heparin: a case series. Ann R Coll Surg Engl 89:309-312

11. Cuculi F, Gurzeler J (2006) What is your diagnosis? Rectus sheath hematoma. Praxis 95:11-12
12. Andereya S, Kalicke T, Hopf KF, Buschmeier M, Muhr G (2003) Serious complication after subcutaneous injection of heparin for prophylaxis of thromboembolism. Case report. Unfallchirurg 106:182-183

13. Pessina I, Bocchia M, Giansante P, Vaghi A (2003) Hematoma of the rectus muscle of abdomen in a patient treated with nadroparin. Recenti Prog Med 94:321-322

14. Luyx C, Vanpee D, Douala C, Gillet JB (2001) Acute dyspnea in a woman with swelling of the left leg treated with low molecular weight heparin. Am J Emerg Med 19:223-224

15. Morau D, Barthelet Y, Spilmann E, d'Athis F (2000) Hematoma of the right rectus abdominis in relation to treatment with lowmolecular-weight heparin. Ann Fr Anesth Rèanim 19:69-70

16. Berna JD, Zuazu I, Madrigal M, Garcia-Medina V, Fernandez C, Guirado F (2000) Conservative treatment of large rectus sheath hematoma in patients undergoing anticoagulant therapy. Abdom Imaging 25:230-234

17. Tsapatsaris NP (1991) Low-dose heparin. A cause of hematoma of rectus abdominis. Arch Intern Med 151:597-599

18. Webb KB, Hadzima S (1987) Hematoma of the rectus abdominis muscle: a complication of subcutaneous heparin therapy. South Med J 80:911-912

19. Zaybak A, Khorshid L (2008) A study on the effect of the duration of subcutaneous heparin injection on bruising and pain. $\mathrm{J}$ Clin Nurs 17:378-385

20. Chan H (2001) Effects of injection duration on site-pain intensity and bruising associated with subcutaneous heparin. J Adv Nurs $35: 882-892$

21. Spahn G (2002) Compliance with self-administration of heparin injections in outpatients. Eur J Trauma 28:104-109

22. Colwell CW, Pullido P, Hardwick ME, Morris BA (2005) Patient compliance with outpatient prophylaxis: an observational study. Orthopedics 28:143-147

23. Watts AC, Howie CR, Simpson AH (2006) Assessment of a self-administration protocol for extended subcutaneous thromboprophylaxis in lower limb arthroplasty. J Bone Joint Surg Br $88: 107-110$

24. Le Gall C, Jacques E, Medjebeur C, Darques L, Briand F, Haddad J, Bleichner G (2006) Low molecular weight heparin selfinjection training: assessment of feasibility, tolerance and economic analysis in emergency departments. Eur J Emerg Med $13: 264-269$

25. Mengiardi S, Goepfert B, Tsakiris DA, Hersberger KE (2009) Pitfalls in patient self-management of subcutaneous drug application: removal of rubber protection caps from ready-to-use syringes. Eur J Clin Pharmacol 65:1061-1062

26. Jorgensen JT, Romsing J, Rasmussen M, Moller-Sonnergaard J, Vang L, Musaeus L (1996) Pain assessment of subcutaneous injections. Ann Pharmacother 30:729-732

27. da Silva AA, Cassiani SH, Optiz SP (2002) Evaluation of the technique of subcutaneous administration of heparin in the development of hematomas. Rev Bras Enferm 55:128-133

28. Lalezari JP, Patel IH, Zhang X, Dorr A, Hawker N, Siddique Z, Kolis SJ, Kinchelow T (2003) Influence of subcutaneous injection site on the steady-state pharmacokinetics of enfuvirtide (T-20) in HIV-1-infected patients. J Clin Virol 28:217-222

29. Frid A, Hardebo JE (1997) The thigh may not be suitable as an injection site for patients self-injecting sumatriptan. Neurology 49:559-561 